\title{
Molecular basis of retinol anti-ageing properties in naturally aged human skin in vivo
}

\author{
Y. Shao, T. He, G. J. Fisher, J. J. Voorhees and T. Quan \\ Department of Dermatology, University of Michigan Medical School, Ann Arbor, 48109, Michigan, MI, U.S.A.
}

Received 22 February 2016, Accepted 25 May 2016

Keywords: extracellular matrix, retinoid, skin ageing, TGF- $\beta$, vascularity

\begin{abstract}
OBJECTIVE: Retinoic acid has been shown to improve the agedappearing skin. However, less is known about the anti-ageing effects of retinol (ROL, vitamin A), a precursor of retinoic acid, in aged human skin in vivo. This study aimed to investigate the molecular basis of ROL anti-ageing properties in naturally aged human skin in vivo.

METHODS: Sun-protected buttock skin $(76 \pm 6$ years old, $n=12)$ was topically treated with $0.4 \%$ ROL and its vehicle for 7 days. The effects of topical ROL on skin epidermis and dermis were evaluated by immunohistochemistry, in situ hybridization, Northern analysis, real-time RT-PCR and Western analysis. Collagen fibrils nanoscale structure and surface topology were analysed by atomic force microscopy.

RESULTS: Topical ROL shows remarkable anti-ageing effects through three major types of skin cells: epidermal keratinocytes, dermal endothelial cells and fibroblasts. Topical ROL significantly increased epidermal thickness by stimulating keratinocytes proliferation and upregulation of c-Jun transcription factor. In addition to epidermal changes, topical ROL significantly improved dermal extracellular matrix (ECM) microenvironment; increasing dermal vascularity by stimulating endothelial cells proliferation and ECM production (type I collagen, fibronectin and elastin) by activating dermal fibroblasts. Topical ROL also stimulates TGF- $\beta /$ CTGF pathway, the major regulator of ECM homeostasis, and thus enriched the deposition of ECM in aged human skin in vivo. $0.4 \%$ topical ROL achieved similar results as seen with topical retinoic acid, the biologically active form of ROL, without causing noticeable signs of retinoid side effects.

CONCLUSION: $0.4 \%$ topical ROL shows remarkable anti-ageing effects through improvement of the homeostasis of epidermis and dermis by stimulating the proliferation of keratinocytes and endothelial cells, and activating dermal fibroblasts. These data provide evidence that $0.4 \%$ topical ROL is a promising and safe treatment to improve the naturally aged human skin.
\end{abstract}

\section{Résumé}

OBJECTIF: L'acide rétinoïque a été montré améliorer l'apparence de la peau âgée. Cependant, on connaît moins les effets anti-vieillissement de rétinol (ROL, de la vitamine A), un précurseur de l'acide

Correspondence: Taihao Quan, Department of Dermatology, University of Michigan Medical School, 1301 Catherine, Medical Science I, Room 6447, Ann Arbor, MI 48109-0609, U.S.A. Tel.: (734) 165 2403; fax: (734) 647 0076; e-mail: thquan@umich.edu rétinoïque, dans la peau humaine in vivo. Cette étude visait à étudier la base moléculaire des propriétés anti-vieillissement du retinol de la peau humaine naturellement vieillie in vivo.

METHODES: La peau des fesses protégée des UV (76 \pm 6 ans, $\mathrm{n}=$ 12) a été traitée par voie topique avec $0,4 \%$ ROL et son véhicule pendant sept jours. Les effets du ROL topique sur l'épiderme et le derme de la peau ont été évalués par immunohistochimie, l'hybridation in situ, l'analyse Northern, RT-PCR en temps réel, et analyse Western. La structure des fibrilles de collagène à l'échelle nanométrique et la topologie de surface ont été analysés par microscopie à force atomique.

RÉSULTATS: ROL par voie topique montre des effets anti-âge remarquables à travers trois grands types de cellules de la peau: les kératinocytes épidermiques, les cellules endothéliales et les fibroblastes dermiques. ROL topique augmente de manière significative l'épaisseur de l'épiderme en stimulant la prolifération des kératinocytes et une régulation positive du facteur de transcription c-Jun. En plus des changements de l'épiderme, le ROL topique améliore significativement le microenvironnement de la matrice extracellulaire (ECM) dermique: augmentation de la vascularisation cutanée par stimulation de la prolifération des cellules endothéliales et la production d'ECM (collagène de type I, la fibronectine et l'élastine), en activant les fibroblastes dermiques. Le ROL topique stimule également la voie TGF- $\beta$ / CTGF, le principal régulateur de l'homéostasie ECM, et donc enrichit le dépôt d'ECM dans la peau âgée humaine in vivo. L'application topique de $0,4 \%$ ROL obtient des résultats similaires à ceux observés avec l'acide rétinoïque topique, la forme biologiquement active de ROL, sans provoquer de signes apparents d'effets secondaires associés aux rétinoïdes.

CONCLUSION: L'application topique de 0,4\% ROL montre des effets remarquables anti-âge grâce à une amélioration de l'homéostasie du derme et l'épiderme, en stimulant la prolifération des kératinocytes et des cellules endothéliales, et l'activation des fibroblastes dermiques. Ces données fournissent la preuve que l'application topique de $0,4 \%$ ROL est un traitement prometteur et sûr pour améliorer la peau humaine vieillie naturellement.

\section{Introduction}

Human skin, like all other organs, undergoes natural ageing process as a consequence of the passage of time [1]. In addition, human skin, unlike other organs, continuously experience harmful stress and damage from environmental sources such as solar UV irradiation [2]. As skin changes are among the most visible signs of ageing, skin is central in the social and visual experience. As 
such, skin appearance has a significant emotional and psychological impact on our life quality. In clinically, aged skin has a significant pathological impact on many age-related skin diseases, notably impaired wound healing [3,4] and promotion of cancer development in elderly $[5,6]$.

Histological and ultrastructural studies have revealed skin undergoes remarkable morphologic changes with ageing, which are primarily characterized by thinner epidermis and dermis with reduced numbers of epidermal keratinocytes and dermal stromal cells, respectively $[7,8]$. This age-related thinning of epidermis and dermis is the major driving force for the aged-appearing skin in elderly. It is well-documented that age-related thinning of the epidermis significantly impairs skin function such as impaired skin barrier function that forms a barrier to prevent water loss and protect against environmental insults. In addition to epidermis, aged skin dermis became thin largely due to loss of collagen, the major structural protein in skin. Age-related thinning of the dermis increases fragility [9, 10], impaired vasculature support [11, 12], poor wound healing [3, 4] and altered tissue microenvironment that promotes cancer development $[5,6]$.

Anti-ageing preventative approaches have been proposed for the purpose of rejuvenating aged human skin and preventing agerelated skin diseases. However, safe and effective therapies to reverse the atrophy of aged skin do not exist currently. Topical retinoic acid has been shown to improve clinical features of aged appearance by reduction in fine wrinkling, increased smoothness and diminished hyperpigmentation [13]. However, the underlying cellular and molecular mechanisms are remaining to be fully elucidated. Moreover, in contrast to retinoic acid, there are limited studies describing the molecular basis of retinol (ROL, vitamin A), a precursor of retinoic acid, anti-ageing effects in human skin in vivo. As human skin has the capacity to convert ROL to its biologically active metabolite retinoic acid, we investigated ROL anti-ageing effects in aged human skin in vivo. Our results demonstrate that topical application of $0.4 \%$ ROL shows remarkable anti-ageing effects in aged human skin through improvement of both epidermis and dermis and enhanced TGF- $\beta$ /CTGF pathway. These data reveal molecular basis of ROL anti-ageing effects and further suggest that topical ROL is a promising and safe treatment to improve naturally aged human skin.

\section{Materials and methods}

Procurement of human tissue samples and retinol treatment in human skin in vivo

All procedures involving human volunteers were approved by the University of Michigan Institutional Review Board, and all volunteers provided written informed consent. Full thickness punch biopsies ( $4 \mathrm{~mm})$ were obtained from aged ( $76 \pm 6$ years) healthy sun-protected buttock skin after topical treatment of vehicle $(70 \%$ ethanal, $30 \%$ polyethylene glycol, and $0.05 \%$ butylated hydroxytoluene) and (ROL, vitamin A, 0.4\%) [14]. Vehicle and ROL were applied topically to sun-protected buttock skin for 7 days under occlusion to prevent loss and exposure to light.

\section{Immunohistology}

Immunohistology was performed as described previously [15]. Briefly, skin OCT-embedded cryo-sections $(7 \mu \mathrm{m})$ were fixed in paraformaldehyde. Subsequently, the slides were incubated for
1 hour at room temperature with normal control serum followed by incubation of primary antibodies against Ki 67, CD31, type I procollagen, fibronectin, TGF-b1 and CTGF/CCN2 (Santa Cruz Biotechnology, Santa Cruz, CA, U.S.A.). To verify antibody specificity, antibodies were incubated with antigenic peptides (Santa Cruz Biotechnology) for $30 \mathrm{~min}$ prior to addition to skin sections. All sections were lightly counterstained with haematoxylin and were mounted with mounting media (Vector Laboratories, Burlingame, CA, U.S.A.). The intensity of positive staining was quantified by computerized image analysis (IMAGE-PRo PLUS software, version 4.1, Media Cybernetics, Rockville, MD, U.S.A.).

\section{RNA isolation and Northern blot analysis}

Total RNA from human skin was extracted with a commercial kit (RNeasy Midi Kit; Qiagen, Chatsworth, CA, U.S.A.) according to the manufacturer's protocol. Samples of total RNA $(30-50 \mu \mathrm{g})$ were resolved by $1.2 \%$ agarose electrophoresis, transferred to nylon membranes and hybridized with TGF- $\beta 1$ [16] and CCN2 CTGF/ CCN2 [17] cDNA probes labelled with $\left[{ }^{32} \mathrm{P}\right] \mathrm{dCTP}$ by random priming. Each blot was stripped and re-hybridized with 36B4 internal control gene transcript to monitor the sample load in each lane. The intensities of each band were quantified by STORM PhosphorImager (Molecular Dynamics, Sunnyvale, CA, U.S.A.) and normalized to the 36B4 gene transcript.

\section{In situ hybridization}

pCDNA3.1 (Invitrogen, Carlsbad, CA, U.S.A.) plasmids containing human TGF- $\beta 1$ and CTGF/CCN2 were linearized with NotI and BamHI. Digoxigenin-containing sense and anti-sense riboprobes were synthesized using SP6 and T7 ribonucleic polymerase. Frozen skin sections $(5 \mu \mathrm{m})$ were mounted, fixed, treated and hybridized as previously described [17]. The hybridization signals were detected immunohistochemically by alkaline phosphatase-conjugated antidigoxigenin antibody.

\section{RNA isolation and quantitative real-time RT-PCR}

Total RNA was extracted from human skin by using a commercial kit (RNeasy mini kit, Qiagen), as previously described [18]. $200 \mathrm{ng}$ total RNA was reverse transcribed using Taqman Reverse Transcription kit (Applied Biosystems, Foster City, CA, U.S.A.). Real-time RTPCR was performed using a Taqman Universal PCR Master Mix kit (Applied Biosystems) and 7700 Sequence Detector (Applied Biosystems). All PCR primers and probes were purchased from Applied Biosystems (Assays-on-Demand ${ }^{\mathrm{TM}}$ Gene Expression Products, Applied Biosystems). Target gene mRNA levels were normalized to the housekeeping gene 36B4 (a ribosomal protein used as an internal control for quantitation) levels as an internal control.

\section{Western blot analysis}

Whole cell proteins were extracted from human skin dermis. The epidermis was removed by cutting off epidermis at a depth of $1 \mathrm{~mm}$ using cryostat. Equal amounts of protein ( $\sim 50 \mu \mathrm{g}$ per lane) were resolved by $6-12 \%$ gradient sodium dodecyl sulphate-polyacrylamide (SDS) gel electrophoresis and transferred to polyvinylidene difluoride membranes. Membranes were then blocked with PBST (Phosphate Buffered Saline with 0.1\% Tween ${ }^{\circledR}$ 20) containing $5 \%$ non-fat milk for one hour at room temperature. Smad7 
primary antibody was generously provided by Dr. Ten Dijke (Leiden University Medical Centrum, Leiden, Netherlands). Primary antibody was incubated for one hour at room temperature. The membranes were washed three times with PBST solution and incubated with rabbit secondary antibody for one hour at room temperature. After washing three times with PBST, the membranes were developed with ECF (Vistra ECF Western blotting system, GE Health Care, Piscataway, NJ, U.S.A.) following the manufacturer's protocol. The membranes were scanned with a STORM PhosphorImager (Molecular Dynamics, Sunnyvale, CA, U.S.A.), the intensities of each band were quantified using ImAgeounant software (GE Health Care, Piscataway, NJ, U.S.A.) and normalized to $\beta$-actin (control).

\section{Atomic force microscopy (AFM) imaging}

Human skin biopsies were embedded in OCT, and cryo-sections $(10 \mu \mathrm{m})$ were attached onto the microscope cover glass $(1.2 \mathrm{~mm}$ diameter, Fisher Scientific Co., Pittsburgh, PA, U.S.A.). These AFM samples were allowed to air dry for at least $24 \mathrm{~h}$ before imaging them to AFM analysis. Images were taken by Dimension Icon AFM system (Bruker-AXS, Santa Barbara, CA, U.S.A.) using a silicon AFM probe (PPP-BSI, force constant $0.01-0.5 \mathrm{Nm}^{-1}$, resonant frequency $12-45 \mathrm{kHz}$, NANOSENSORS ${ }^{\mathrm{TM}}$, Switzerland). AFM was conducted at the Electron Microbeam Analysis Laboratory (EMAL), University of Michigan College of Engineering, and analyzed using Nanoscope Analysis software (Nanoscope Analysis v120R1sr3, Bruker-AXS, Santa Barbara, CA, U.S.A.).

\section{Statistical analysis}

Comparisons between groups were determined with the Student's ttest. All $P$ values are two-tailed, and considered significant when $P<0.05$.

\section{Results}

Topical ROL increases epidermal thickness and dermal vascularity by proliferation of epidermal keratinocytes and dermal endothelial cells, respectively, in aged human skin in vivo

As thin epidermis is a characteristic feature of aged human skin, we found that epidermal thickness was greatly improved following ROL treatment (Fig. 1A). Quantitative morphometric analyses revealed that epidermal thickness was increased 2.1-fold by ROL treatment (Fig. 1B). Consistent with thickened epidermis, keratinocyte proliferation, assessed by Ki67 immunostaining (Fig. 1C upper right panel), was increased 12 -fold by ROL treatment (Fig. 1D). Interestingly, in addition to epidermal changes, we noticed topical ROL markedly increased the proliferation of dermal stromal cells (Fig. 1C lower right panel, Fig. 1E). No Ki67 positive cell was observed in vehicletreated aged skin dermis (Fig. 1C lower left panel, Fig. 1E). Ki67 positive staining cells were particularly strong in papillary dermis. To confirm the identity of proliferating dermal stromal cells, we performed double-label immunofluorescence staining. As papillary dermis contains rich vascular networks, we found the majority of Ki67 positive cells were stained with CD31, a marker of endothelial cells (Fig. 1F). This result was further supported by increased prominence of blood vessels, confirmed by CD31 immunostaining (Fig. 1G). Quantitative analyses revealed that blood vessel formation was increased 3.8-fold in ROL-treated aged human skin (Fig. 1H). Together, these data indicate that topical ROL increases epidermal thickness and dermal angiogenesis by stimulating the proliferation of epidermal keratinocytes and dermal endothelial cells, respectively, in aged human skin in vivo.

ROL improves dermal ECM microenvironment by stimulating the expression of type I collagen, fibronectin and tropoelastin in aged human skin in vivo

As thin dermis is a prominent feature of aged skin dermis, we analyzed the expression of major ECM proteins by immunohistochemistry. Type I procollagen is the precursor of mature type I collagen fibrils. Compared with vehicle-treated aged skin, ROL treatment increased type I collagen staining, the major structural protein in skin (Fig. 2A, right panels). Type I procollagen positive staining was particularly strong in epidermal and dermal junction (Fig. 1A lower right panel). Quantitative analysis indicated that the amount of staining was increased three-fold by topical ROL treatment (Fig. 2B). In addition to type I collagen, topical ROL markedly increased the expression of fibronectin (FN) (Fig. 2C, right panels) and tropoelastin (Fig. 2E, right panels). Quantification indicated that fibronectin (Fig. 2D) and elastin (Fig. 2F) staining were increased 2.2-fold and four-fold, respectively. These data indicate that topical ROL improves dermal ECM microenvironment by stimulating the major ECM proteins in aged human skin in vivo.

\section{Elevated epidermal-specific c-Jun transcription factor by topical ROL in aged human skin in vivo}

Next, we explored the potential mechanisms by which topical ROL stimulates keratinocytes proliferation in aged human skin. It has been reported that AP-1 transcription factor plays a major role in keratinocytes proliferation in response to growth factors, cytokines and various stimuli [2]. As AP-1 complex largely comprised of cJun/c-fos, we examined the expression c-Jun and c-Fos in aged human skin after topical ROL treatment. We found that topical ROL markedly increased epidermal-specific c-Jun protein positive staining (Fig. 3A right panel) along with significant epidermal thickness. No c-Jun positive cell was observed in the dermis. Quantitative analyses revealed that c-Jun positive staining was increased 2.3-fold in ROL-treated aged human skin (Fig. 3A right panel). In contrast, no change of c-Fos protein expression was observed by topical ROL treatment (Fig. 3B). Western analysis further confirmed that whereas c-fos was constitutively expressed (Fig. 3D), cJun protein was elevated three-fold (Fig. 3C) by topical ROL treatment. These data suggest that topical ROL elevates epidermal-specific c-Jun transcription factor, which in turn drives epidermal keratinocytes proliferation in aged human skin in vivo.

\section{Elevated TGF- $\beta$ /CTGF pathway by topical ROL in aged human skin in vivo}

Next, we explored the potential mechanisms by which ROL stimulates dermal ECM production in aged human skin. We first examined whether topical ROL is able to activate dermal fibroblasts, the major ECM-producing cells in skin, by staining with HSP47, the marker of fibroblast activation [19]. We observed that topical ROL markedly increased HSP47 positive staining, suggesting the activation of dermal fibroblasts in aged human skin in vivo. (Fig 4A, right panel). Quantitative analyses revealed that HSP47-positive staining was increased 3.8-fold in ROL-treated aged human skin (Fig. 4A right panel). 
A

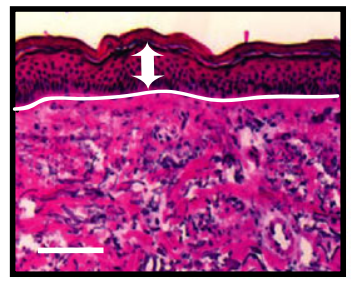

CTRL

C

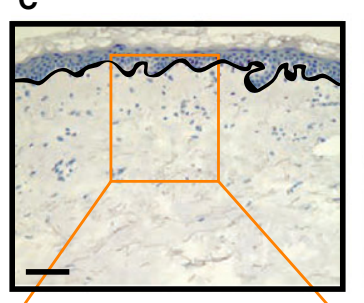

Figure 1 Topical ROL increases epidermal thickness and dermal vascularity by proliferation of epidermal keratinocytes and dermal endothelial cells, respectively, in aged human skin in vivo. OCT-embedded skin sections $(7 \mu \mathrm{m}) \quad$ were obtained from aged (76 \pm 6 years) healthy sun-protected buttock skin after topical treatment of vehicle and $0.4 \%$ retinol for 7 days. (A) H\&E staining. Representative images of twelve individuals $(n=12)$. White arrows indicate epidermal thickness. Bars $=100 \mu \mathrm{m}$. (B) Quantification of epidermal thickness $(\mu \mathrm{m})$. (C) Ki67 immunostaining. 3.0× enlargement of the boxed region is shown to lower panels. Bars $=100 \mu \mathrm{m}$. Red arrows indicate Ki67 positive cells. Representative images of twelve individuals $(n=12)$. Quantification of epidermal (D) and dermal (E) Ki 67 immunostaining. (F) Ki67 and CD31 co-immunofluorescence staining. Skin sections were coimmunofluorescence stained with Ki67 and CD31, a marker of endothelial cells. Arrows indicate double stained cells. Representative of five individuals. $\operatorname{Bar}=50 \mu \mathrm{m}$. (G) CD31 immunostaining. Representative images of twelve individuals $(n=12)$. Bar $=100 \mu \mathrm{m}$. $(\mathrm{H})$ Quantification of CD31 immunostaining. All immunostainings were quantified by computerized image analysis (Image-pro Plus software, version 4.1, Media Cybernetics, MD) and data are expressed as mean \pm SEM, $* P<0.05$. $n=12$.

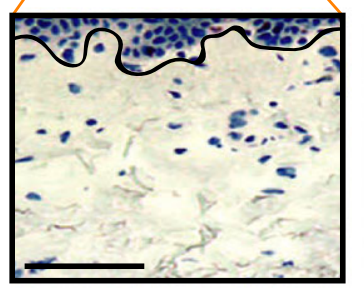

CTRL

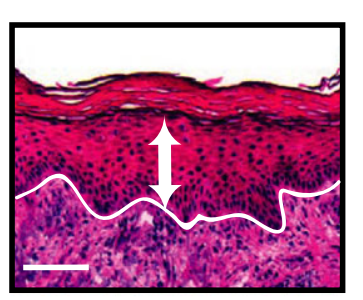

B

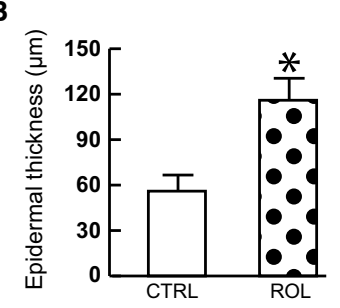

ROL
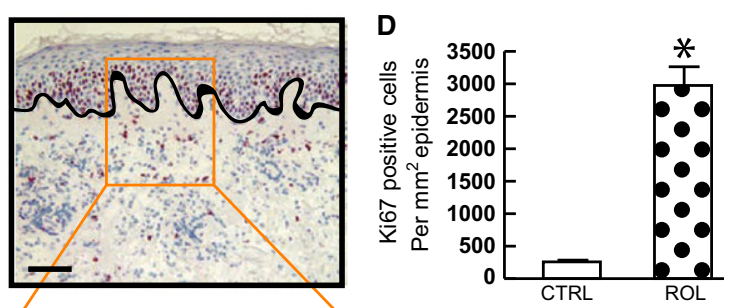

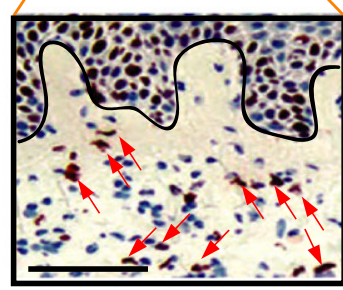

$\mathrm{ROL}$

F

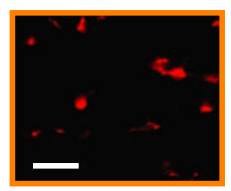

Ki67

G

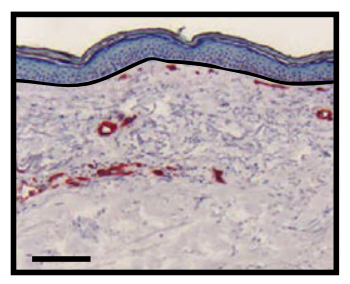

CTRL

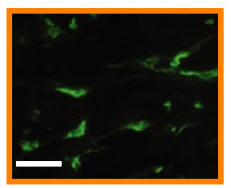

$\mathrm{CD} 31$

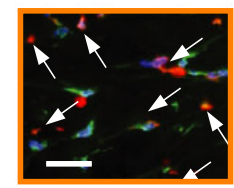

Merge

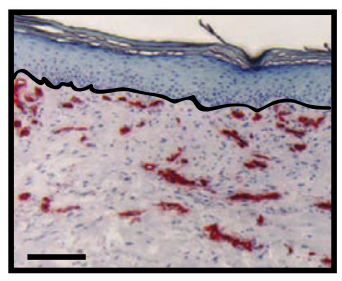

$\mathrm{ROL}$
It has been well-documented that activated dermal fibroblasts increase ECM production through TGF- $\beta$ pathway, the major regulator of ECM production [20]. Therefore, we investigated the possible involvement of TGF- $\beta$ pathway in increased ECM production by topical ROL in aged human skin. To test this possibility, we examined the effect of topical ROL on TGF- $\beta$ pathway components such as TGF- $\beta$ receptors, ligands and Smads proteins. Interestingly, topical ROL significantly upregulated TGF- $\beta 1$ mRNA and down-regulated the inhibitory Smad7, whereas other TGF- $\beta$ pathway components remained unchanged (Fig. 4B). To further confirm these results, we performed In situ hybridization and immunostaining of TGF- $\beta 1$ and its downstream mediator connective tissue growth factor (CTGF). In situ hybridization indicated that intense staining of TGF- $\beta 1$ (Fig. $4 \mathrm{C}$ ) and CTGF/CCN2 (Fig. 4D). Quantification indicated that TGF- $\beta 1$ (Fig. $4 \mathrm{C}$ right panel) and CTGF (Fig. 4D right panel) dermal staining were increased 3.1-fold and 2.8-fold, respectively, following topical ROL treatment. Northern analysis further confirmed that TGF- $\beta 1$ (Fig. 4E) and CTGF/CCN2 (Fig 4F) transcription were increased 2.6-fold and 3.8-fold, respectively, after topical ROL treatment. Consistent with mRNA expression, immunostaining of TGF- $\beta 1$ (Fig. 4G) and CTGF/CCN2 (Fig. 4H) were increased 2.7-fold and 
A

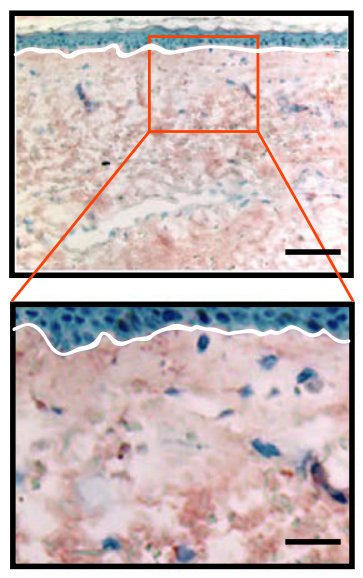

CTRL

C
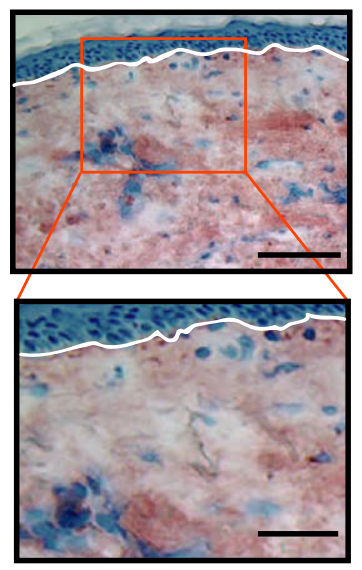

CTRL

E

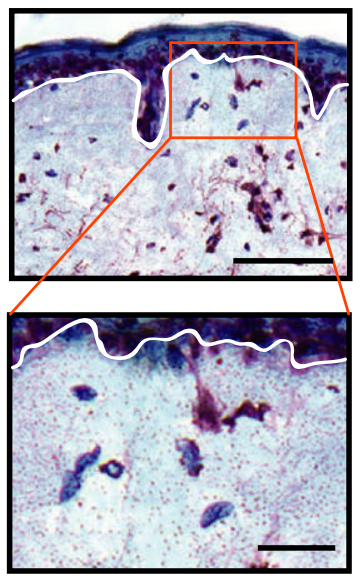

CTRL

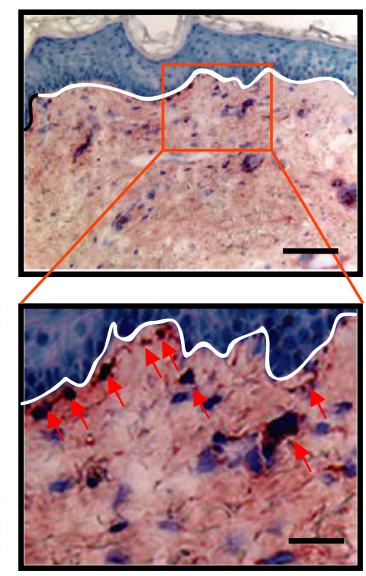

ROL
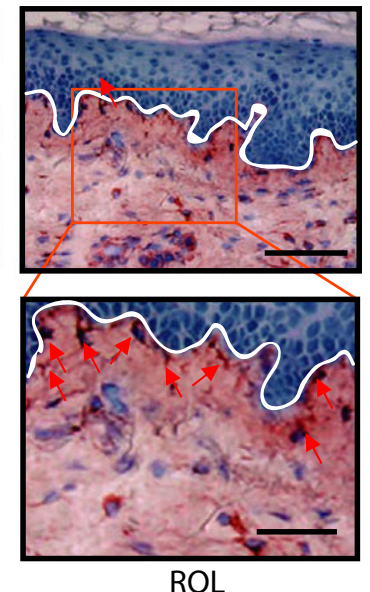

ROL
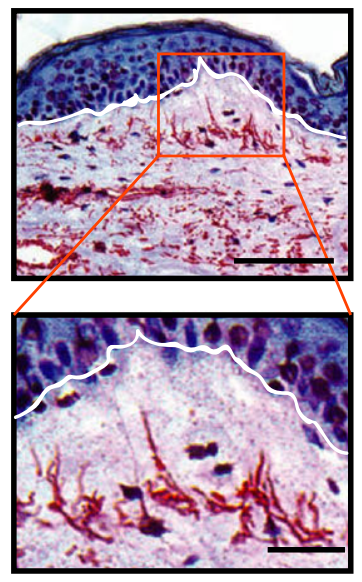

ROL
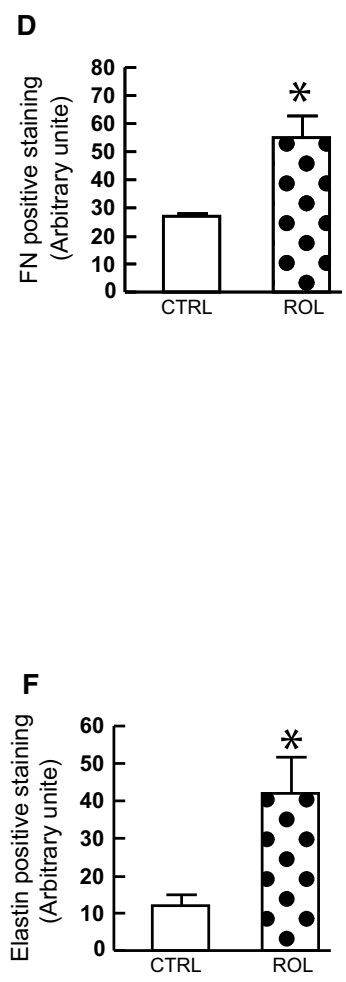

Figure 2 ROL improves dermal ECM microenvironment in aged human skin in vivo. OCTembedded skin sections $(7 \mu \mathrm{m})$ were obtained from aged (76 \pm 6 years) healthy sun-protected buttock skin after topical treatment of vehicle and $0.4 \%$ retinol for 7 days. (A) Type I procollagen immunostaining. $3 \times$ enlargement of the boxed region is shown to lower panels. Representative images of twelve individuals $(n=12)$. Arrows indicate positive cells. Bars $=100 \mu \mathrm{m}$. (B) Quantification of type I procollagen. (C) Fibronectin immunostaining. $2.5 \times$ enlargement of the boxed region is shown to lower panels. Representative images of twelve individuals $(n=12)$. Arrows indicate positive cells. Bars $=100 \mu \mathrm{m}$. (D) Quantification of fibronectin. (E) Tropoelastin immunostaining. $3.0 \times$ enlargement of the boxed region is shown to lower panels. Representative images of twelve individuals $(n=12)$. Bars $=100 \mu \mathrm{m}$. (F) Quantification of tropoelastin. All immunostainings were quantified by computerized image analysis (Image-pro Plus software, version 4.1, Media Cybernetics, MD) and data are expressed as mean \pm SEM, $* P<0.05 . n=12$. 2.8-fold, respectively, after topical ROL treatment. We further confirmed that ROL treatment significantly reduced Smad7 mRNA (Fig. 4I) and protein (Fig. 4J), a potent inhibitor of TGF- $\beta$ signalling. These data suggest that topical ROL stimulates dermal fibroblasts ECM production through upregulation of TGF- $\beta /$ CTGF pathway in aged human skin. 
Figure 3 Elevated epidermal-specific c-Jun transcription factor by topical ROL in aged human skin in vivo. OCT-embedded skin sections $(7 \mu \mathrm{m})$ were obtained from aged (76 \pm 6 years) healthy sun-protected buttock skin after topical treatment of vehicle and $0.4 \%$ retinol for 7 days. (A) c-Jun immunostaining. Representative images of twelve individuals $(n=12)$. Bars $=100 \mu \mathrm{m}$. (B) c-Fos immunostaining. Representative images of twelve individuals $\quad(n=12) . \quad$ Bars $=100 \mu \mathrm{m} . \quad$ All immunostainings were quantified by computerized image analysis (Image-pro Plus software, version 4.1, Media Cybernetics, MD) and data are expressed as mean \pm SEM, $* P<0.05$. $n=12$. (C) c-Jun protein. (D) c-Fos protein. Protein levels were determined by Western analysis. Protein levels were normalized to $\beta$ actin (loading control). Insets show representative Western blots. Mean \pm SEM, $n=6$, $* P<0.05$.
A

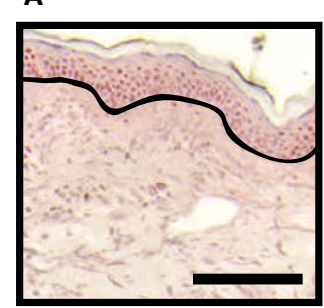

CTRL

B

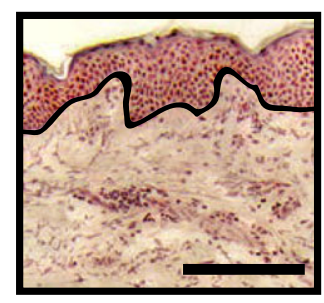

CTRL

C
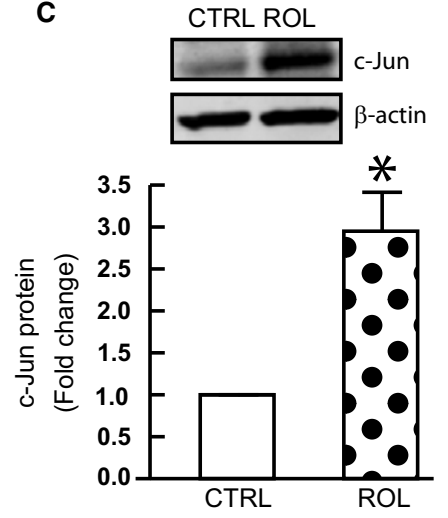

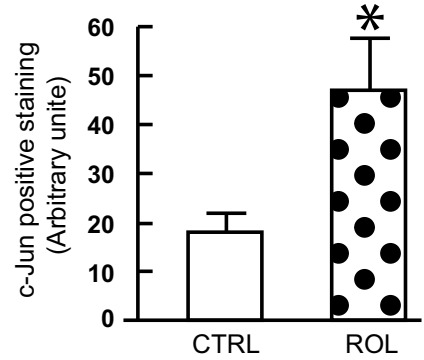

ROL

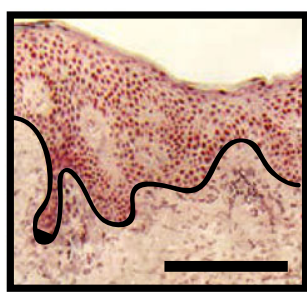

ROL

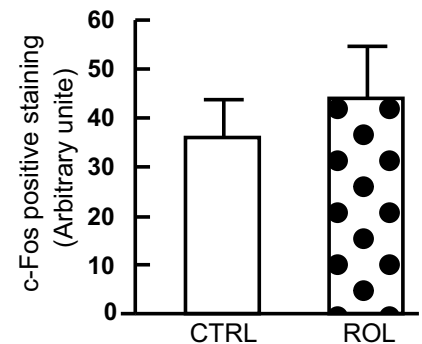

D

CTRL ROL
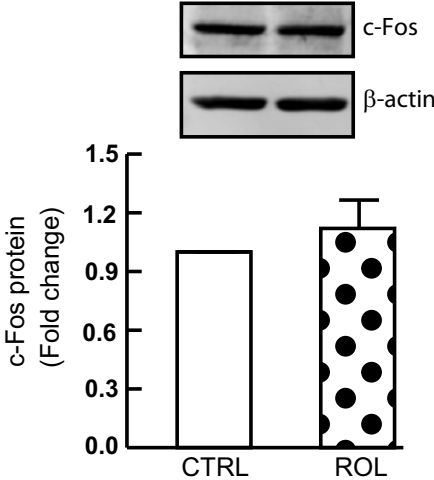

Topical ROL increases the deposition of mature collagen in aged human skin in vivo

Having found that ROL is able to stimulate collagen production, we next assessed the collagen fibrils nanoscale structure and surface topology by (AFM). In vehicle-treated aged skin, collagen fibrils appeared disorganized and fragmented (Fig. 5A, left panel). However, topical ROL-treated aged skin revealed highly organized, dense bundles of collagen fibrils, with characteristic banded structure (D-spacing) representing the staggered alignment of individual collagen molecules within fibrils (Fig. 5A, right panel). Threedimensional topographical AFM images further indicated that vehicle-treated aged skin dermis was much rougher and uneven (Fig. 5B, left panel). In contract, ROL-treated aged skin dermis was smooth and flattened (Fig. 5B, right panel). Quantitative analysis of AFM data indicated that the average roughness (a measure of fibril organization) of dermal collagen fibrils in ROL-treated aged skin is $48 \%$ less than in vehicle-treated aged skin dermis (Fig. 5B, $16 \mathrm{~nm}$ vs. $31 \mathrm{~nm}$ ). These findings indicate that ROL treatment stimulates synthesis of procollagen, which is processed into mature collagen in aged human skin.

\section{Discussion}

Retinoids is the term used for the group of vitamin A derivatives [21]. Retinoic acid is the biologically active form of vitamin A which is also known as (ROL), a precursor to retinoic acid. Human skin has the capacity to convert ROL to its biologically active metabolite retinoic acid. When topically applied to human skin, it penetrates and is sequentially converted to retinaldehyde and then to retinoic acid. One important benefit of topical ROL is that compared with retinoic acid, ROL induces fewer signs of side effects, which is characterized by erythema, scaling, dryness and pruritis $[22,23]$. Our results indicate that although a much higher concentration of ROL $(0.4 \%)$ is needed to achieve similar results as seen with topical retinoic acid, ROL induced the same histological changes (epidermal hyperplasia and dermal ECM production) as retinoic acid without causing retinoids irritation, the most common 
A

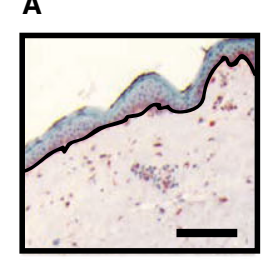

CTRL

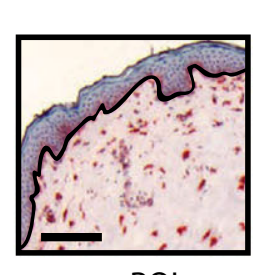

ROL

B

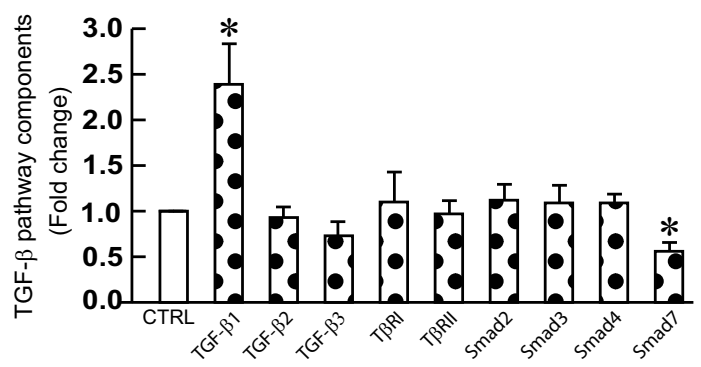

C

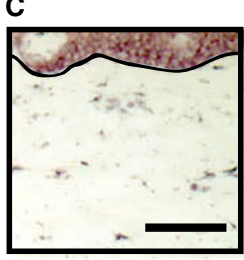

CTRL

D

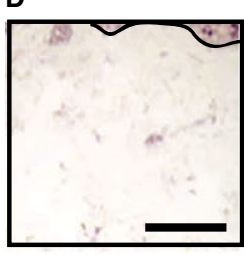

CTRL

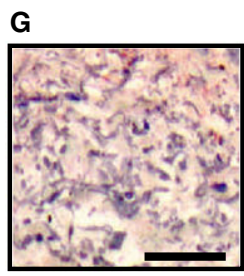

CTRL

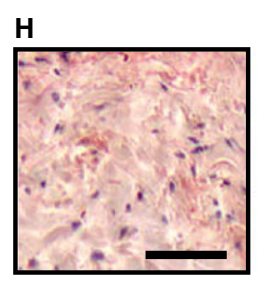

CTRL

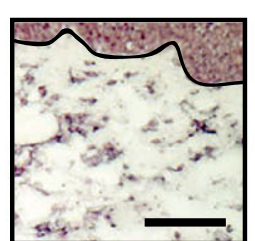

ROL

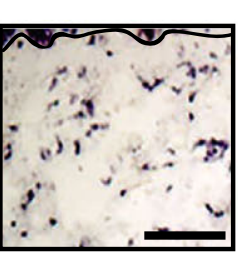

ROL

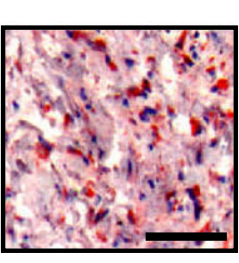

ROL

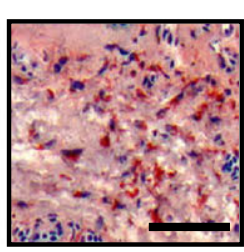

ROL
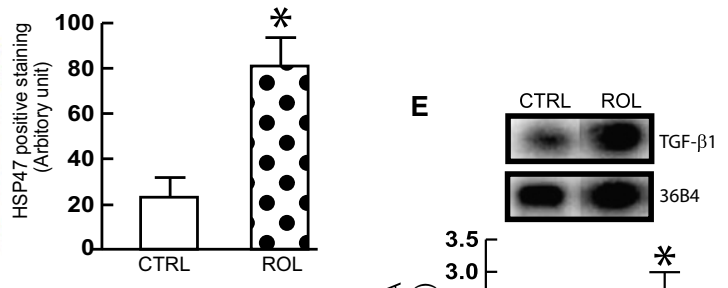

政 2.5

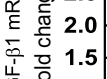

殅说 1.5

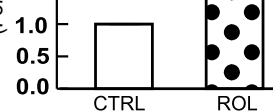

F $\quad$ CTRL ROL
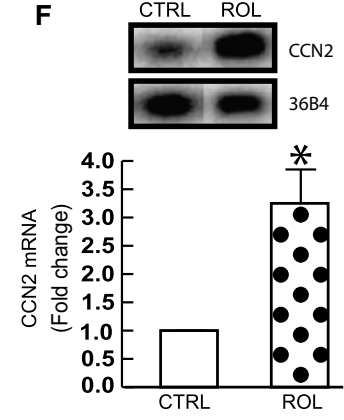

I

CTRL ROL

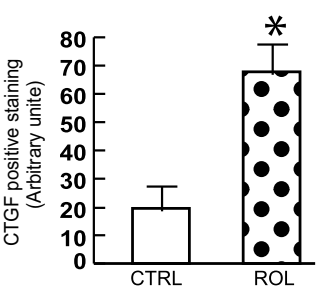

Smad7

3684
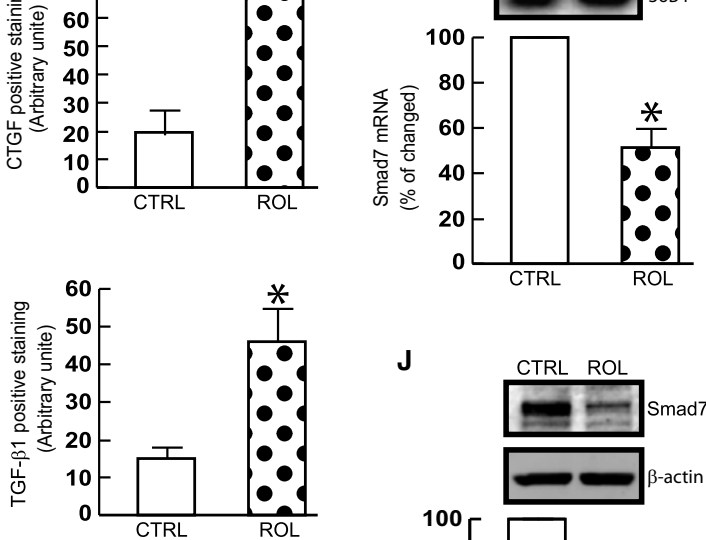

$\mathbf{J}$

CTRL ROL

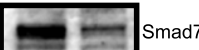

$\beta$-actin

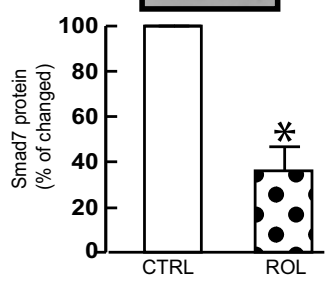


Figure 4 Topical ROL stimulates TGF- $\beta /$ CTGF pathway, the major regulator of ECM homeostasis, in aged human skin in vivo. OCT-embedded skin sections $(7 \mu \mathrm{m})$ were obtained from aged ( $76 \pm 6$ years) healthy sun-protected buttock skin after topical treatment of vehicle and $0.4 \%$ retinol for 7 days. (A) HSP 47 immunostaining. Representative images of twelve individuals $(n=12)$. Bars $=100 \mu \mathrm{m}$. (B) TGF- $\beta$ pathway components mRNA levels. Total RNA was prepared from human skin samples. mRNA levels were determined by real-time RT-PCR. mRNA levels were normalized to $36 \mathrm{~B} 4$ (internal housekeeping gene control). Mean \pm SEM, $n=12, * P<0.05$. (C) TGF- $\beta 1$ (D) CTGF/CCN2 in situ hybridization. Representative images of twelve individuals $(n=12)$. Bars $=100 \mu$ m. $(\mathrm{E})$ TGF- $\beta 1$ (F) CTGF/CCN2 Northern analysis. The intensities were quantified and normalized using 36B4 as loading control. Insets show representative Northern blots. Data are expressed as mean \pm SEM, ${ }^{*} P<0.05 . n=12$. (G) TGF- $\beta 1$ (H) CTGF/CCN2 immunostaining. Representative images of twelve individuals $(n=12$ ). Bars $=100 \mu \mathrm{m}$. (I) Smad7 Northern analysis. The intensities were quantified and normalized using 36B4 as loading control. Inset shows representative Northern blots. Data are expressed as mean \pm SEM, $* P<0.05 . n=12$. (J) Smad7 protein. Smad7 protein levels were determined by Western analysis. Protein levels were normalized to $\beta$-actin (loading control). Insets show representative Western blots. Mean \pm SEM, $n=6$, $* P<0.05$. All positive staining was quantified by computerized image analysis (Image-pro Plus software, version 4.1, Media Cybernetics, MD). Data are expressed as mean \pm SEM, ${ }^{*} P<0.05 . n=12$.

side effect of retinoids. Mechanistically, it appears that topical ROL increases epidermal thickness by stimulating keratinocytes proliferation, which involves epidermal-specific upregulation of c-Jun transcription factor. Topical ROL also significantly elevates ECM production, which involves enhanced TGF- $\beta /$ CTGF pathway in the dermis. Additionally, topical ROL significantly increased dermal vascularity which may have a significant impact on the homeostasis of epidermis and dermis.

\section{A}

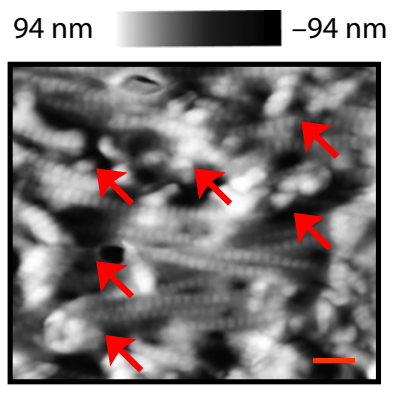

CTRL

B

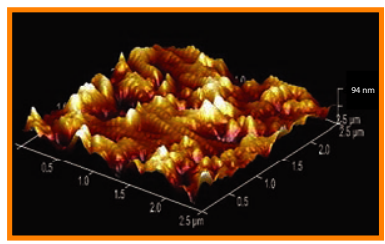

CTRL

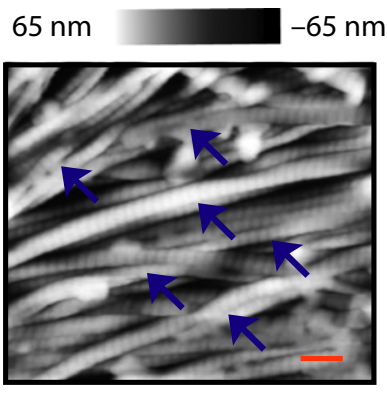

$\mathrm{ROL}$

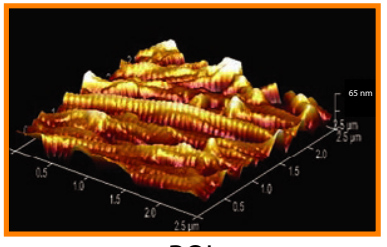

$\mathrm{ROL}$

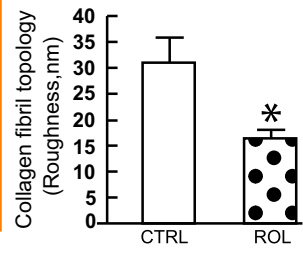

C

Figure 5 Deposition of mature collagen is increased by topical ROL in aged human skin in vivo. OCT-embedded skin sections $(7 \mu \mathrm{m})$ were obtained from aged $(76 \pm 6$ years $)$ healthy sun-protected buttock skin after topical treatment of vehicle and $0.4 \%$ retinol for 7 days. (A) Nanoscale collagen fibrils were imaged by AFM. Representative AFM images were shown. $n=12$. The blue arrows indicate intact collagen fibrils and red arrow heads indicate damaged collagen fibrils. (B) Three-dimensional collagen fibrils. Dermal roughness was analyzed using Nanoscope Analysis software (Nanoscope_Analysis_v120R1sr3, Bruker-AXS, Santa Barbara, CA). All results are expressed as the mean \pm SEM, $n=12, \quad * P<0.05$. Bars $=100 \mathrm{~nm}$. (C) Proposed model for topical ROL exerts anti-ageing effects in aged human skin by proliferation of epidermal keratinocytes and dermal endothelial cells and activation of dermal fibroblasts (see Discussion for details).
Topical $0.4 \%$ ROL

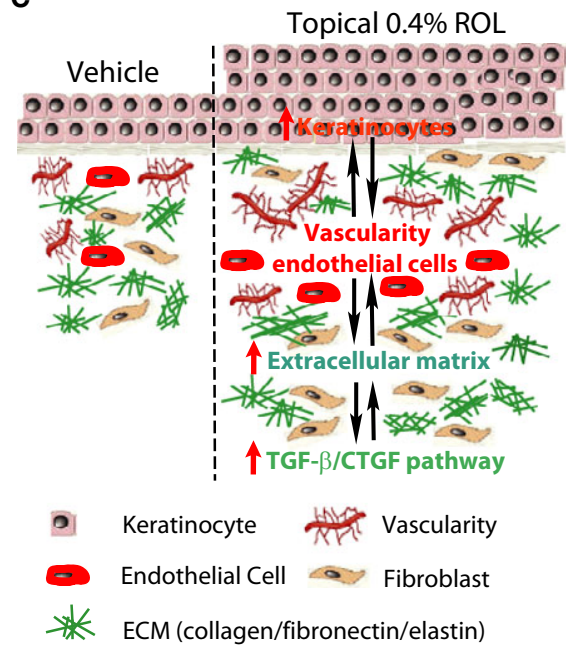


Although topical retinoids have been widely used in the treatment of skin ageing, the precise mechanism(s) by which retinoids lead to visible improvement in aged skin is not fully understood. One significant finding of our study is that topical ROL improves aged dermal ECM microenvironment through enhancement of TGF- $\beta /$ CTGF pathway in the dermis, the principle regulator of ECM homeostasis [20] ROL is able to enhance TGF- $\beta /$ CTGF pathway through two independent pathways: increases of the expression of TGF- $\beta 1 / \mathrm{CTGF}$ and suppression of inhibitory Smad7 of TGF- $\beta$ signalling (Fig. 4). In primary human skin fibroblasts, the major ECM-producing cells in skin, the expression of major ECM proteins is regulated by TGF- $\beta /$ CTGF pathway $[7,20]$. For example, neutralization of endogenous TGF- $\beta$ or knockdown of CTGF substantially reduced the expression of type I procollagen and fibronectin mRNA and protein. In contrast, overexpression of inhibitory Smad7, which is significantly reduced by topical ROL (Fig. 4B, I, J), abolished TGF- $\beta$ or CTGF stimulation of ECM production. Importantly, we have previously reported that TGF- $\beta /$ CTGF pathway is significantly reduced in dermal fibroblasts, in aged (80+years) human skin in vivo $[7,20,24,25]$. This impaired TGF- $\beta /$ CTGF pathway in aged human skin is largely caused by reduced expression of TGF- $\beta 1$ and CTGF. As impaired TGF- $\beta /$ CTGF pathway significantly contributes to thinned dermis, our data suggest that the ability of topical ROL to improve aged skin dermis involves enhanced TGF- $\beta$ /CTGF pathway.

Reduced vasculature and epidermal thinning contribute significantly to skin fragility and impaired wound healing in aged skin $[26,27]$. AP-1 transcription factor plays a major role in keratinocytes proliferation [13]. We found that whereas c-Fos is constitutively expressed in human skin, topical ROL elevates epidermal-specific c-Jun transcription factor. These data suggest that topical ROL may stimulate keratinocytes proliferation by epidermal-specific upregulation c-Jun transcription factor in aged human skin. Our study added another new piece of information that topical ROL improves dermal microenvironment through not only stimulation of ECM production, but also expansion of vasculature by proliferation of endothelial cells in aged human skin (Fig. 1C, F, G). A reduction of the cutaneous vasculature has been reported in aged human skin [27]. Increased vascularity of the dermis by topical ROL could increase skin blood flow and provide a more suitable microenvironment for the homeostasis of skin epidermal and dermal homeostasis. In contrast, it is also conceivable that proliferation of epidermal keratinocytes and restoration of ECM production by topical ROL may provide a more favourable microenvironment for the proliferation of endothelial cells and dermal vasculature. Indeed, epidermal keratinocytes are important source of vascular endothelial growth factor (VGEF), a potent angiogenic factor [27]. Additionally, it has been reported that enhanced dermal ECM production resulted in stimulation of endothelial cell proliferation [19]. Clearly, additional studies are warranted to uncover the precise molecular mechanism(s) by which topical ROL stimulates the proliferation of epidermal keratinocytes and dermal endothelial cells in aged human skin. The molecular mechanisms by which retinoids improve aged human skin have been difficult to investigate largely due to lack of appropriate in vitro models. For example, cells in both the epidermis and dermis contain all the proteins and receptors that mediate the biological effects of vitamin A metabolites in the skin. However, primary keratinocytes or dermal fibroblasts are minimally responsive to RA treatment in monolayer culture $[14,28]$. This lack of responsiveness is due, at least in part, to low levels of nuclear retinoid receptors, which mediate expression of retinoid-regulated genes [29, 30]. Skin equivalent cultures could be a useful model to investigate retinoids regulation of collagen homeostasis [14]. Skin equivalent cultures are composed of stratified, differentiated keratinocytes (model epidermis) on top of a type I collagen lattice, in which dermal fibroblasts are embedded (model dermis). Treatment of skin equivalent cultures with retinoic acid significantly increased the number of keratinocyte layers and dermal response, similar to the effect of topical application of RA to human skin in vivo. These data suggest that skin equivalent cultures could be a useful model for investigating mechanisms by which retinoids improve aged-appearing skin in human.

Together, our findings extend current knowledge of retinoids anti-ageing mechanisms in aged human skin. We propose a working model for the molecular basis of ROL anti-ageing properties in aged human skin (Fig. 5C). Topical application of $0.4 \%$ ROL to aged human skin leads to remarkable skin changes in both epidermis and dermis through affecting three major types of skin cells, epidermal keratinocytes, dermal endothelial cells and fibroblasts. Topical ROL significantly increases epidermal thickness by stimulating epidermal keratinocytes proliferation, which involves c-Jun transcription factor, a major driving force for keratinocyte proliferation. In addition to epidermal changes, topical ROL significantly improves dermal ECM microenvironment; increasing dermal blood vessel formation by stimulating endothelial cells proliferation and ECM production by activating fibroblasts. Topical ROL also stimulates TGF- $\beta /$ CTGF pathway, the major regulator of ECM homeostasis, and thus increased the deposition of mature collagen in aged human skin in vivo.

Increased dermal vasculatures could increase skin blood flow and provide a more active microenvironment for both epidermal keratinocyte proliferation and dermal fibroblasts activation. On the other hand, the proliferation of epidermal keratinocytes may also promote dermal blood vessel growth through stimulation of VEGF expression [27]. Additionally, the restoration of dermal ECM may provide a better, more permissive environment for the proliferation of dermal endothelial cells and epidermal keratinocytes, and activation of dermal fibroblasts (TGF- $\beta /$ CTGF pathway) [19]. We propose that coupling of the proliferation of keratinocytes and endothelial cells, and dermal fibroblasts activation forms a selfenforcing environment, which might explain the remarkable antiageing effects of ROL in aged human skin. Proposed model extends current understanding of the ROL anti-ageing mechanisms in human skin.

\section{Acknowledgments}

We thank Suzan Rehbine for the procurement of tissue specimens and Diane Fiolek for graphic and administrative assistance. We thank Electron Microbeam Analysis Laboratory (EMAL), University of Michigan College of Engineering for AFM. This work was supported by the National Institute of Health (AG019364 to GJ Fisher and T Quan; ES014697 to T Quan), Dermatology Foundation Research grant (to T Quan), and a grant from the Johnson \&Johnson Corporation. 


\section{References}

1. Quan, T., Skin connective tissue aging and dermal fibroblasts. In: Dermal Fibroblasts: Histological Perspectives, Characterization and Role in Disease (Xiaojing, Bai, ed.), pp. 3155. Nova Biomedical, New York (2013).

2. Fisher, G.J., Kang, S., Varani, J., BataCsorgo, Z., Wan, Y., Datta, S. and Voorhees, J.J. Mechanisms of photoaging and chronological skin aging. Arch. Dermatol. 138, 1462-1470 (2002).

3. Thomas, D.R. and Burkemper, N.M. Aging skin and wound healing. Clin. Geriatr. Med. 29, $x i-x x$ (2013).

4. Worley, C.A. Aging skin and wound healing. Dermatol. Nurs. 18, 265-266 (2006).

5. Bissell, M.J. and Hines, W.C. Why don't we get more cancer? A proposed role of the microenvironment in restraining cancer progression. Nat. Med. 17, 320-329 (2011).

6. Bissell, M.J., Kenny, P.A. and Radisky, D.C. Microenvironmental regulators of tissue structure and function also regulate tumor induction and progression: the role of extracellular matrix and its degrading enzymes. Cold Spring Harb. Symp. Quant. Biol. 70, 343-356 (2005).

7. Quan, T. and Fisher, G.J. Role of age-associated alterations of the dermal extracellular matrix microenvironment in human skin aging: a mini-review. Gerontology 61, 427434 (2015).

8. Yaar, M., Eller, M.S. and Gilchrest, B.A. Fifty years of skin aging. J. Investig. Dermatol. Symp. Proc. 7, 51-58 (2002).

9. Smith, J.G., Jr Davidson, E.A., Sams, W.M. Jr and Clark, R.D. Alterations in human dermal connective tissue with age and chronic sun damage. J. Invest. Dermatol. 39, 347350 (1962).

10. Uitto, J. and Bernstein, E.F. Molecular mechanisms of cutaneous aging: connective tissue alterations in the dermis. J. Investig. Dermatol. Symp. Proc 3, 41-44 (1998).

11. Jacob, M.P. Extracellular matrix remodeling and matrix metalloproteinases in the vascular wall during aging and in pathological conditions. Biomed. Pharmacother. 57, 195202 (2003).
12. Marastoni, S., Ligresti, G., Lorenzon, E., Colombatti, A. and Mongiat, M. Extracellular matrix: a matter of life and death. Connect. Tissue Res. 49, 203-206 (2008).

13. Fisher, G.J., Talwar, H.S., Lin, J. and Voorhees, J.J. Molecular mechanisms of photoaging in human skin in vivo and their prevention by all-trans retinoic acid. Photochem. Photobiol. 69, 154-157 (1999).

14. Quan, T., Qin, Z., Shao, Y., Xu, Y., Voorhees, J.J. and Fisher, G.J. Retinoids suppress cysteine-rich protein 61 (CCN1), a negative regulator of collagen homeostasis, in skin equivalent cultures and aged human skin in vivo. Exp. Dermatol. 20, 572-576 (2011).

15. Quan, C., Cho, M.K., Shao, Y., Mianecki, L.E., Liao, E., Perry, D. and Quan, T. Dermal fibroblast expression of stromal cell-derived factor-1 (SDF-1) promotes epidermal keratinocyte proliferation in normal and diseased skin. Protein Cell 6, 890-903 (2015).

16. Quan, T., He, T., Kang, S., Voorhees, J.J. and Fisher, G.J. Ultraviolet irradiation alters transforming growth factor beta/smad pathway in human skin in vivo. J. Invest. Dermatol. 119, 499-506 (2002).

17. Quan, T., He, T., Kang, S., Voorhees, J.J. and Fisher, G.J. Connective tissue growth factor: expression in human skin in vivo and inhibition by ultraviolet irradiation. $J$. Invest. Dermatol. 118, 402-408 (2002).

18. Quan, C., Cho, M.K., Perry, D. and Quan, T. Age-associated reduction of cell spreading induces mitochondrial DNA common deletion by oxidative stress in human skin dermal fibroblasts: implication for human skin connective tissue aging. J. Biomed. Sci. 22, 62 (2015).

19. Quan, T., Wang, F., Shao, Y. et al. Enhancing structural support of the dermal microenvironment activates fibroblasts, endothelial cells, and keratinocytes in aged human skin in vivo. J. Invest. Dermatol. 133, 658-667 (2013)

20. Quan, T., Shao, Y., He, T., Voorhees, J.J. and Fisher, G.J. Reduced expression of connective tissue growth factor (CTGF/CCN2) mediates collagen loss in chronologically aged human skin. J. Invest. Dermatol. 130 415-424 (2010).

21. Blomhoff, R. Transport and metabolism of vitamin A. Nutr. Rev. 52, S13-S23 (1994).

22. Mukherjee, S., Date, A., Patravale, V., Korting, H.C., Roeder, A. and Weindl, G. Retinoids in the treatment of skin aging: an overview of clinical efficacy and safety. Clin. Interv. Aging 1, 327-348 (2006).

23. Darlenski, R., Surber, C. and Fluhr, J.W Topical retinoids in the management of photodamaged skin: from theory to evidencebased practical approach. Br. J. Dermatol. 163, 1157-1165 (2010).

24. Quan, T., He, T., Shao, Y., Lin, L., Kang, S., Voorhees, J.J. and Fisher, G.J. Elevated cysteine-rich 61 mediates aberrant collagen homeostasis in chronologically aged and photoaged human skin. Am. J. Pathol. 169, 482-490 (2006).

25. Fisher, G.J., Shao, Y., He, T., Qin, Z., Perry, D., Voorhees, J.J. and Quan, T. Reduction of fibroblast size/mechanical force down-regulates TGF-beta type II receptor: implications for human skin aging. Aging Cell 15, 67-76 (2016).

26. Zouboulis, C.C. and Makrantonaki, E. Clinical aspects and molecular diagnostics of skin aging. Clin. Dermatol. 29, 3-14 (2011).

27. Chung, J.H. and Eun, H.C. Angiogenesis in skin aging and photoaging. J. Dermatol. 34, 593-600 (2007).

28. Elder, J.T., Astrom, A., Pettersson, U. et al. Differential regulation of retinoic acid receptors and binding proteins in human skin. J. Invest. Dermatol. 98, 673-679 (1992).

29. Di, W., Li, X.Y., Datta, S. et al. Keratinocyte-specific retinoid regulation of human cellular retinoic acid binding protein-II (hCRABPII) gene promoter requires an evolutionarily conserved DR1 retinoic acid-responsive element. J. Invest. Dermatol. 111, 1109-1115 (1998).

30. Fisher, G.J., Talwar, H.S., Xiao, J.H. et al. Immunological identification and functional quantitation of retinoic acid and retinoid $X$ receptor proteins in human skin. J. Biol. Chem. 269, 20629-20635 (1994). 